\title{
Le pouvoir de vote dans les établissements publics de coopération intercommunale de la Martinique et de la Guadeloupe
}

The voting power in the inter-communal council of Martinique and Guadeloupe

Ibrahima Dia et Éric Kamwa

\section{(2) OpenEdition \\ Journals}

Édition électronique

URL : http://journals.openedition.org/ei/6546

DOI : 10.4000/ei.6546

ISSN : 2553-1891

Éditeur

Association Économie et Institutions

Référence électronique

Ibrahima Dia et Éric Kamwa, «Le pouvoir de vote dans les établissements publics de coopération intercommunale de la Martinique et de la Guadeloupe », Économie et institutions [En ligne], 28 | 2020, mis en ligne le 29 juin 2020, consulté le 10 août 2020. URL : http://journals.openedition.org/ei/6546 ; DOI : https://doi.org/10.4000/ei.6546

Ce document a été généré automatiquement le 10 août 2020.

Revue Économie et institutions 


\section{Le pouvoir de vote dans les établissements publics de coopération intercommunale de la Martinique et de la Guadeloupe}

The voting power in the inter-communal council of Martinique and Guadeloupe

Ibrahima Dia et Éric Kamwa

\section{Introduction}

1 À la suite de la loi $\mathrm{n}^{\circ} 2010-1563$ du 16 décembre 2010 relative à la réforme des collectivités territoriales, la loi $\mathrm{n}^{\circ}$ 2015-991 du 7 août 2015 relative à la nouvelle organisation territoriale de la République (Loi NOTRe) a profondément modifié le paysage intercommunal en France. La Martinique et la Guadeloupe, tout comme les autres départements d'outre-mer, n'ont pas été impactées par le vaste mouvement de fusions et de recompositions des périmètres intercommunaux résultant de la mise en œuvre de la Loi NOTRe ${ }^{1}$. Les établissements publics de coopération intercommunale (EPCI) en Martinique et en Guadeloupe s'illustrent par leur étendue : les communes ont en moyenne une superficie de $51 \mathrm{~km}^{2}$ en Guadeloupe et $33 \mathrm{~km}^{2}$ en Martinique contre une moyenne nationale de $19 \mathrm{~km}^{2}$. On note aussi qu'avec de fortes densités de population (plus de deux fois supérieures à la moyenne nationale), $83 \%$ des EPCI de la Guadeloupe regroupent plus de 50000 habitants (contre seulement $22 \%$ à l'échelle nationale) et l'intégralité des EPCI de Martinique comptent plus de 100000 habitants (contre seulement $9 \%$ à l'échelle de la France); entre 2008 et 2013, la Martinique a connu une baisse (de $6 \%$ ) de sa population, alors que la population de la Guadeloupe est restée quasi stable sur cette période. Les faits que nous venons d'exposer soulèvent des interrogations : la répartition du nombre de délégués au sein de chacun des EPCI de ces deux territoires est-elle équitable? Existe-t-il une différence entre le "pouvoir apparent » des communes (mesuré sur la base du nombre de délégués) et le « pouvoir 
réel " au sein des EPCI ? Comme nous le verrons dans la suite de cet article, des réponses à ces questions ont été apportées dans la littérature concernant plusieurs EPCI en France métropolitaine. À notre connaissance, aucune étude n'a abordé ces questionnements dans le cas des territoires d'outre-mer. D'où l'intérêt de se pencher de plus près sur la répartition du nombre de délégués au sein des EPCI de la Martinique et de la Guadeloupe en vue de mettre en exergue ou d'infirmer l'existence de possibles distorsions entre la distribution des indices de pouvoir de vote et celle des pourcentages de délégués.

2 Selon le Rapport CNFPT (2015) ${ }^{2}$, l'expression «intercommunalité » désigne les différentes formes ${ }^{3}$ de coopération existant entre les communes. Le regroupement de communes au sein d'EPCI répond à deux objectifs différents : la gestion commune de certains services publics locaux ou la réalisation d'équipements locaux de manière à réaliser des économies d'échelles, et la conduite collective de projets de développement local.

La recherche de performance et d'efficacité de l'offre des biens publics se trouve au cœur de la formation des structures de coopération intercommunale. En coopérant, les communes tentent d'apporter des solutions aux problèmes de financement des biens et services publics au sein d'un territoire élargi. En France, la première loi sur la coopération, datant de 1890, donnait le droit aux communes de se regrouper en SIVU et SIVOM $^{5}$ dans le but de mutualiser l'offre de certains biens publics. Les années 1990 ont marqué un tournant dans la formation des structures de coopération intercommunale. Un certain nombre de lois et de réformes incitant les communes à former des établissements publics de coopération intercommunale (EPCI) ont été élaborées. Sans être exhaustif, nous pouvons citer la loi nº 92-125 du 6 février 1992 relative à l'administration territoriale de la République instituant la création des communautés de communes (CDC) ; la loi no 99-586 du 12 juillet 1999 (loi Chevènement) relative au renforcement et à la simplification de la coopération intercommunale instituant la création des communautés d'agglomération (CA); la loi $\mathrm{n}^{\circ} 2004-809 \mathrm{du}$ 13 août 2004 relative aux responsabilités et libertés locales facilitant le fonctionnement de l'intercommunalité et encourageant les fusions de communautés ainsi que le partage de services entre communes et communautés. La loi n ${ }^{\circ}$ 2010-1563 du 16 décembre 2010 relative à la réforme des collectivités territoriales oblige toutes les communes à rejoindre une intercommunalité à fiscalité propre avant 2014. Enfin, la loi no 2015-991 du 7 août 2015 relative à la nouvelle organisation territoriale de la République (Loi NOTRe) renforce les compétences des EPCI. L'un des objectifs de ces lois était de réduire l'intensité de la concurrence fiscale entre communes et de faire face au manque de revenus financiers de certaines communes.

4 Les départements d'outre-mer, notamment la Martinique et la Guadeloupe, ont également dû aborder les problématiques de l'intercommunalité tant sur la pertinence des regroupements des communes que sur la définition de l'intérêt communautaire dans l'exercice du partage des compétences locales. Depuis 2014, les 34 communes de la Martinique se sont regroupées en trois communautés d'agglomération ${ }^{6}$ : la communauté d'agglomération du centre de la Martinique (CACEM), la communauté d'agglomération de l'Espace sud Martinique (CAESM) et la communauté d'agglomération du Pays Nord Martinique (CAP Nord). Les 32 communes de la Guadeloupe se sont regroupées en cinq communautés d'agglomérations ${ }^{7}$ et une communauté de communes: la communauté d'agglomération du Sud Grande-Terre 
(CASGT), la communauté d'agglomération La Riviera du Levant, la communauté d'agglomération du Nord Grande-Terre (CANGT), la communauté d'agglomération du Nord Basse-Terre (CANBT), la communauté d'agglomération Grand Sud Caraïbe $(\mathrm{CAGSC})^{8}$ et la communauté de communes de Marie-Galante (CCMG).

5 L'objectif de la loi de 2010 était non seulement d'obliger toutes les communes à rejoindre une structure intercommunale au $1^{\mathrm{er}}$ janvier 2014, mais également de réduire le nombre de délégués dans les EPCI et de définir les critères d'allocation du nombre de délégués entre les communes membres des EPCI ${ }^{9}$. L'allocation du nombre de délégués constitue en elle-même l'un des problèmes du développement de l'intercommunalité. Cette allocation tenant compte de la population pour fixer le nombre de délégués par commune, le pouvoir de chaque commune dans le processus de décision collective serait proportionnel à sa population. Ceci peut donner lieu à une perte de pouvoir des petites communes par rapport aux grandes, car les modes de prise de décision dans les EPCI (à la majorité simple ou des deux tiers) se traduisent souvent par une dilution et/ ou un déficit de pouvoir. Ces effets peuvent être mis en exergue à l'aide des outils qu'offre la théorie des jeux: les indices de pouvoir. Ces outils sont utilisés dans la littérature pour mesurer le pouvoir des agents engagés dans un processus de décision collective, leur capacité à être décisif. L'objectif de cet article est d'étudier la répartition du pouvoir au sein des communautés de communes de la Martinique et de la Guadeloupe au moyen des indices de pouvoir, qui ici permettront de mesurer le contrôle de chaque commune sur les décisions prises collectivement au sein des EPCI.

De la vaste classe des indices qui existent dans la littérature, notre analyse portera sur deux d'entre eux: l'indice de Shapley-Shubik et l'indice de Banzhaf-Coleman ${ }^{10}$. Ces deux indices seront formellement définis dans la Section 3. Il convient de noter que, sur un plan empirique, les indices de pouvoir ont été largement utilisés dans la littérature et pour des applications diverses. On peut ainsi noter, entre autres, les travaux portant sur le Conseil de l'Union européenne: Bobay (2001, 2004), Felsenthal et Machover (2001,2003), Garrett et Tsebelis (1999), Herne et Nurmi (1993), Leech (2002), Leech et Aziz (2010) ; ceux portant sur la Loi LRU ${ }^{11}$ (Barthelemy, Beraud \& Martin, 2008, 2009) ; les élections présidentielles américaines (Barthelemy \& Martin, 2007) et le partage de pouvoir au Liban (Diss \& Zouache, 2015 ; Diss \& Steffen, 2018). Pour d'autres applications, le lecteur peut se référer aux travaux de Felsenthal et Machover (1998), Holler et Nurmi (2013).

7 En ce qui concerne l'application des indices de pouvoir aux EPCI françaises, on peut citer sans être exhaustif les travaux de Bisson, Bonnet et Lepelley (2004), Bonnet et Lepelley (2001), Barthelemy et Martin (2007) ainsi que Abidi, Le Prince et Merlin (2019). Bonnet et Lepelley (2001) étudient la répartition du pouvoir de vote dans les EPCI de Basse-Normandie à l'aide des indices de Shapley-Shubik et de Banzhaf-Coleman. Ils mettent en évidence que pour ces EPCI il n'existe pas de distorsions importantes entre la répartition des poids des communes, mesurée par leur nombre de délégués, et leurs indices de pouvoir. Bisson, Bonnet et Lepelley (2004) prolongent l'analyse de Bonnet et Lepelley (2001) en prenant en compte la possible absence d'associations préférentielles entre les communes et tentent de proposer une ou plusieurs distributions des délégués au sein du conseil communautaire qui permettent d'atteindre la structure de pouvoir choisie. Barthelemy et Martin (2007) étudient la répartition des sièges au sein des structures intercommunales du département du Val-d'Oise en comparant, à partir des indices de pouvoir de Shapley-Shubik et de Banzhaf, la distribution observée de sièges à une distribution théorique obtenue par la méthode de Webster. Ils concluent que les 
répartitions de sièges observées ne sont pas les bonnes et que le choix du nombre de sièges à pourvoir est souvent malvenu. Abidi, Le Prince et Merlin (2019) analysent l'équité dans la répartition des délégués (l'égalisation du pouvoir de vote) dans $377 \mathrm{EPCI}$ avant et après la loi du 16 décembre 2010. Ces auteurs arrivent à la conclusion que la répartition des sièges avant 2010 était assez équitable, un nombre important de grandes municipalités acceptant de contrôler moins de délégués qu'elles n'auraient pu le faire.

8 À notre connaissance, aucun travail académique n'a à ce jour analysé les EPCI d'outremer en termes de pouvoir de vote des communes. Le présent article tente de combler ce vide. La suite de notre travail s'organise de la manière suivante : dans la Section 2, nous présentons les règles qui fixent le nombre de délégués dans les EPCI et la manière dont s'opère la répartition entre les communes membres. Nous présentons la répartition des délégués au sein des EPCI de la Martinique et de la Guadeloupe. Dans la Section 3 nous présentons de manière formelle les indices de Banzhaf-Coleman et de Shapley-Shubik. Nous appliquons ces indices aux EPCI de la Martinique et de la Guadeloupe, ce qui nous permet de mettre en lumière les distorsions qui existent d'une part entre le pouvoir des communes et leur poids démographique, d'autre part entre le pouvoir des communes et leur poids en termes de délégués. Notons que nous aurions $\mathrm{pu}$ restreindre notre analyse à un seul des deux indices; le souci d'aboutir à des conclusions robustes justifie le recours simultané aux deux indices.

\section{La répartition du nombre de délégués dans les $\mathrm{EPCI}$}

En référence à l'article L.5210-1 du Code général des collectivités territoriales, la coopération intercommunale se fonde sur la libre volonté des communes d'élaborer des projets communs de développement au sein de périmètres de solidarité. L'établissement public de coopération intercommunale est créé par un arrêté préfectoral avec des statuts qui mentionnent la liste des communes membres, le nombre de délégués par communes et ses compétences.

\subsection{La répartition du nombre de délégués dans les EPCI}

10 La répartition et le nombre de sièges de conseillers communautaires sont établis selon les dispositions de l'article L.5211-6-1 du Code général des collectivités territoriales, modifié par la loi $\mathrm{n}^{\circ}$ 2017-257 du 28 février 2017, article 75. La loi de 2010 attribue à chaque structure intercommunale un nombre de délégués communautaires selon la taille de sa population comme on peut le voir dans la Table 1.

Table 1. Répartition du nombre de sièges selon la population

\begin{tabular}{|l|l|}
\hline Population de l'EPCI & Nombre de sièges \\
\hline De moins de 3500 habitants & 16 \\
\hline De 3500 à 4999 habitants & 18 \\
\hline De 5000 à 9999 habitants & 22 \\
\hline
\end{tabular}




\begin{tabular}{|l|l|}
\hline De 10000 à 19999 habitants & 26 \\
\hline De 20000 à 29999 habitants & 30 \\
\hline De 30000 à 39999 habitants & 34 \\
\hline De 40000 à 49999 habitants & 38 \\
\hline De 50000 à 74999 habitants & 40 \\
\hline De 75000 à 99999 habitants & 42 \\
\hline De 100000 à 149999 habitants & 48 \\
\hline De 150000 à 199999 habitants & 56 \\
\hline De 200000 à 249999 habitants & 64 \\
\hline De 250000 à 349999 habitants & 72 \\
\hline De 350000 à 499999 habitants & 80 \\
\hline De 500000 à 699999 habitants & 90 \\
\hline De 700000 à 1000000 habitants & 100 \\
\hline Plus de 1000000 habitants & 130 \\
\hline
\end{tabular}

Source : élaboré par les auteurs à partir de l'article 9 de la loi n²010-1563 du 16 décembre 2010 relative à la réforme des collectivités territoriales

11 Cette répartition, qui se veut proportionnelle, se fait sur la base de la méthode de Jefferson qui intervient une fois fixé le nombre de sièges à répartir. Cette méthode procède de la manière suivante :

a. on commence par calculer le « diviseur » qui indique le nombre de personnes correspondant à un élu. Le diviseur s'obtient en calculant le rapport entre la population totale de l'EPCI et le nombre de sièges à répartir.

\section{diviseur $=\frac{\text { population totale de l'EPCI }}{\text { nombre de sièges à répartir d'après la Table } 1}$}

a. Ensuite, on détermine le «quota» pour chaque commune de l'EPCI; il correspond au rapport entre la population de la commune et le diviseur.

$$
\text { quota }=\frac{\text { population de la commune }}{\text { diviseur }}
$$

12 Le quota pouvant être un nombre décimal, il est arrondi à l'entier inférieur, c'est-à-dire que seule sa partie entière est retenue et constitue le nombre de sièges alloués à la commune. Si tous les sièges n'ont pas été alloués, les sièges restants sont attribués un à 
un sur la base de la règle de la plus forte moyenne. Cette moyenne correspond au rapport suivant :

population de la commune nombre de sièges déjà obtenus +1

Si au terme du processus une commune n'a obtenu aucun siège, un siège lui est automatiquement attribué. De même, si une commune se retrouve avec plus de la moitié des sièges, la loi recommande de ne lui conserver que cette moitié et de redistribuer les sièges supplémentaires sur la base de la plus forte moyenne. Ainsi, à la fin du processus, le nombre total de sièges alloués pourrait être supérieur à celui recommandé dans la Table 1.

a. Notons toutefois que la loi de 2010 permet aussi aux EPCI de pouvoir déterminer le nombre de délégués des communes membres sur la base d'un arrangement ; ceci pourvu qu'au moins deux tiers des délégués municipaux appartenant à des municipalités représentant au moins cinquante pour cent de la population totale de l'EPCI soient d'accord sur le principe. Dans ce cas de figure, la loi permet d'obtenir dix pour cent de sièges supplémentaires par rapport à ceux de la Table 1.

13 Notons qu'en dehors de la méthode de Jefferson, il existe d'autres méthodes de répartition des sièges. Pour un aperçu des différentes méthodes de répartition des sièges et des enjeux de cette répartition en lien avec la taille de la population, le lecteur peut, par exemple, se référer aux travaux de Balinski et Young (2001), Barthelemy et Martin (2011), Felsenthal et Machover $(1998,2003)$. Il convient à toute fin utile de mentionner qu'il n'existe pas de "meilleure » règle de répartition dans la mesure où chacune des règles qui existent présente des qualités et des défauts (limites) tant d'ordre conceptuel, normatif que pratique. L'ouvrage de Balinski et Young (2001) pourra mieux éclairer le lecteur sur cette observation.

\subsection{Répartition des sièges dans les communautés d'agglomération martiniquaises}

14 La répartition du nombre de délégués dans chacun des trois EPCI de la Martinique est consignée dans la Table 2 (en annexe). Notons que le nombre de délégués de la CACEM obéit aux recommandations de la Table 1. Pour Cap Nord, le nombre de sièges aurait été de 48 si l'on s'en était tenu à la Table 1 ; six sièges supplémentaires ont été ajoutés sur la base du processus décrit dans le paragraphe précédent, portant ainsi le nombre de délégués à 54 . Le cas de la CAESM mérite une attention particulière. Étant donné la population totale de la CAESM et d'après la Table 1, le nombre de délégués aurait été de 48 mais, dans les faits, il est de 37. Ce nombre ne respecte aucune des règles définies plus haut et cela nous amène à nous interroger sur ce qu'aurait été la répartition des sièges dans la CAESM si l'on s'en était tenu au processus décrit précédemment. Il ressort de la Table 2 que, comparativement à la distribution des sièges faite sur la base de 48 sièges comme le recommande la Table 1 , la répartition actuelle pénalise quasiment toutes les communes à l'exception de la commune des Anses-d'Arlet pour qui la répartition actuelle est favorable ; les deux répartitions ne diffèrent pas en ce qui concerne le nombre de délégués des communes des Trois-Ilets et de Sainte-Anne. 
Table 4. Tests de Khi-deux de similarité des distributions entre le pourcentage de la population et celui du nombre de délégués

\begin{tabular}{|l|l|l|l|l|}
\hline & Communauté d'agglomération & Khi2 & $\mathbf{p}$-value & Conclusion \\
\hline \multirow{5}{*}{ Martinique } & CACEM & 12,00 & 0,213 & Non \\
\cline { 2 - 5 } & CAESM & 24,00 & 0,347 & Non \\
\cline { 2 - 5 } & CAP Nord & 108,00 & 0,189 & Non \\
\hline \multirow{5}{*}{ Guadeloupe } & CA Nord Grande-Terre & 20,00 & 0,220 & Non \\
\cline { 2 - 6 } & CA Nord Basse-Terre & 6,00 & 0,199 & Non \\
\cline { 2 - 6 } & La Riviera du Levant & 12,00 & 0,213 & Non \\
\cline { 2 - 6 } & CA & 30,00 & 0,224 & Non \\
\cline { 2 - 6 } & CA Grand Sud Caraïbe & 55,00 & 0,291 & Non \\
\cline { 2 - 6 } & CC de Marie-Galante & 6,00 & 0,199 & Non \\
\hline
\end{tabular}

Source : calculs des auteurs à l'aide du logiciel SPSS 20.0

Dans la Table 4, nous avons effectué un test de Khi-deux de similarité entre la distribution du pourcentage de la population et celle du nombre de délégués au sein de chacun des EPCI de la Martinique. Comme le montre la Table 4, pour chacun des EPCI, il n'existe aucune similarité entre les distributions; de plus les p-valeurs sont assez élevées (plus de $18 \%$ ) dans chacun des cas. Ceci semble traduire une forme de sur/sousreprésentation de certaines communes comparativement aux autres.

\subsection{Répartition des sièges dans les communautés d'agglomération guadeloupéennes}

La Table 3 (en annexe) nous donne un aperçu de la répartition actuelle du nombre de délégués dans chacun des EPCI de la Guadeloupe. Comme on peut le constater, à l'exception de la communauté d'agglomération du Nord Basse-Terre, le nombre de délégués dans les autres EPCI diffère de celui que recommande la Table 1, et la répartition actuelle des délégués entre les communes n'est pas conforme à la règle de répartition décrite plus haut. Dans de tels cas, nous avons calculé (voir la dernière colonne de la Table 3) la répartition qui aurait été obtenue sur la base des recommandations de la Table 1 et de la règle de répartition indiquée dans la loi. La communauté d'agglomération Cap excellence compte 50 délégués alors que la Table 1 en recommande 48. Ceci reste légal dans la mesure où la loi accorde une possibilité de marge de $10 \%$. Avec cet ajout de deux délégués, la répartition entre les trois communes reste conforme à la règle. La communauté de communes de Marie-Galante dispose actuellement de 16 délégués, soit un nombre bien inférieur à celui de 26 délégués que recommande le principe de la population; cette différence impacte fortement le 
rapport de force entre les communes en termes de poids (pourcentage de délégués). Dans le cas de la communauté d'agglomération Grand Sud Caraïbe, le nombre de délégués obéit aux recommandations de la Table 1 mais la répartition actuelle des délégués entre les communes n'est pas conforme à la règle de répartition: la répartition actuelle octroie un siège supplémentaire à la commune de Baillif alors qu'elle en prive la commune de Saint-Claude.

17 Le test de Khi-deux de la Table 4 témoigne du fait qu'il n'existe pas de similarité entre la distribution du pourcentage de la population et celle du nombre de délégués au sein de chacun des EPCI de la Guadeloupe; de plus, les p-valeurs sont assez élevées (plus de $19 \%)$ dans chacun des cas. Ceci traduit, comme dans le cas de la Martinique, que certaines communes sont sur/sous-représentées comparativement aux autres.

\section{La mesure du pouvoir dans les EPCI}

Dans cette section, nous mesurons le pouvoir des communes dans chacun des EPCI inclus dans notre analyse. Avant de nous concentrer sur cette application, intéressonsnous aux outils développés dans ce sens par la théorie des jeux.

\subsection{Les indices de pouvoir de Shapley-Shubik et de Banzhaf- Coleman}

Les outils les plus utilisés dans la littérature pour mesurer le pouvoir des agents engagés dans un processus de décision collective sont issus de la théorie des jeux coopératifs. Il est supposé que chaque agent, encore appelé joueur (ici, les communes), vote pour ou contre ${ }^{12}$ une proposition qui est présentée à l'ensemble des décideurs, et le résultat dépend de la règle de décision prédéterminée. La règle de décision permet de déterminer le(s) sous-ensemble(s) d'agents (coalitions) qui conduisent à l'adoption de la proposition. Dans cet article, nous considérons les systèmes de vote dans lesquels les joueurs (communes) peuvent avoir des poids différents, et la proposition est adoptée si la somme des poids des joueurs qui votent pour est au moins égale à un certain seuil fixé à l'avance : on parle de jeux de vote pondéré. Soit que le jeu implique $n$ joueurs dotés chacun d'un poids $w_{i}(i=1,2, \ldots, n)$ (et que le seuil minimal pour que la proposition soit adoptée est . Le système de vote est défini par la liste $\left[q ; w_{1}, w_{2}, \ldots, w_{n}\right]$. On note par $N$ l'ensemble des $n$ joueurs; tout $S$ sous-ensemble de $N$ est appelé « coalition ». Étant donné $q$, une coalition sera dite gagnante si la somme des poids des agents membres de cette coalition est supérieure ou égale à $q$. De prime abord, on serait tenté de croire que les poids des joueurs correspondent à leur pouvoir ; il n'en est rien. La théorie des jeux dispose de plusieurs indices qui permettent de mesurer le pouvoir des joueurs (Straffin, 1977 ; Pajala, Meskanen \& Kause, 2002 ; Andjiga, Chantreuil \& Lepelley, 2003). Dans cet article, nous nous intéressons à deux de ces indices: l'indice de Shapley-Shubik (Shapley \& Shubik, 1954) et l'indice de Banzhaf-Coleman (Banzhaf, 1965 ; Coleman, 1971).

L'indice de Shapley-Shubik du joueur $i$ se calcule selon la formule suivante : 


$$
S S(i)=\sum_{S \subseteq N} \frac{(s-1) !(n-s) !}{n !}[v(S)-v(S-\{i\})]
$$

Où $s$ désigne le cardinal de $S ; v(S-\{i\})$ est la coalition $S$ sans le joueur $i ; v(S)$ désigne la fonction caractéristique du système de vote définie comme suit :

$$
v(S)=\left\{\begin{array}{lr}
1 & \text { si } \sum_{i \in S} w_{i} \geq q \\
0 & \text { sinon }
\end{array}\right.
$$

$$
S \subseteq N
$$

L'indice de Shapley-Shubik tient compte de l'ordre des joueurs dans la formation des coalitions, ce qui n'est pas le cas de l'indice de Banzhaf-Coleman. Cet indice suppose que le pouvoir d'un joueur est proportionnel au nombre de coalitions dans lesquelles le retrait de ce joueur transforme cette coalition en perdante. L'indice de BanzhafColeman du joueur $i$ se calcule selon la formule suivante :

$$
B C(i)=\frac{\sum_{S \subseteq N}[v(S)-v(S-\{i\})]}{\sum_{j \in N} \sum_{S \subseteq N}[v(S)-v(S-\{j\})]}
$$

Notons que d'après la loi de la racine carrée de Penrose $(1946,1952)$, en appliquant la loi des grands nombres, l'indice de Banzhaf-Coleman d'un joueur (ici, une commune) est approximativement proportionnel à son nombre de délégués; il s'ensuit qu'il y a de fortes chances que le pouvoir égal des électeurs (habitants) soit atteint, au moins approximativement, avec une répartition des sièges proportionnelle à la racine carrée de la population.

Étant donné leurs formulations, l'indice de Shapley-Shubik et celui de BanzhafColeman donnent le plus souvent des résultats différents. Pajala, Meskanen et Kause (2002) ont créé un site internet où ils présentent de manière synthétique la plupart des indices de pouvoir qui existent dans la littérature. Ce site offre également une interface permettant de calculer les indices de pouvoir ${ }^{13}$. Ainsi, nous avons eu recours à ce site pour le calcul de nos indices. Concernant nos tests statistiques, nous avons utilisé le logiciel SPSS 20.

Nous pouvons désormais nous intéresser à la mesure du pouvoir des communes de nos EPCI. Nous procédons en supposant que la décision collective au sein des EPCI est prise selon les règles suivantes : la majorité simple et la majorité des deux tiers. D'après le CGCT $^{14}$ (art. L.2121-17, L.2121-20 et L.2121-21), les délibérations au sein des conseils communautaires se font à la majorité absolue des membres présents. La règle de la majorité des deux tiers est requise en cas de modification de la répartition des sièges ou de l'évolution de l'intérêt communautaire des compétences (art. L.5721-2-1). Nos calculs supposent que tous les délégués d'une commune votent dans le même sens. Nous admettons que cette hypothèse est forte car abstraction est faite des aspects psychologiques et politiques des associations préférentielles entre les communes. 


\subsection{Le pouvoir des communes dans les $\mathrm{EPCI}$ martiniquais} Martinique sont consignés dans la Table 5 (en annexe). De la Table 5 nous déduisons que :

- dans le cas de la CACEM, la commune de Fort-de France apparaît comme incontournable car selon les indices et la règle de décision, elle détient au moins $50 \%$ du pouvoir de décision. Selon les termes de la théorie des jeux, la commune de Fort-de-France est un joueur "pivot ». La CACEM est la seule EPCI de la Martinique avec un joueur pivot. Lorsque l'on passe de la règle de la majorité simple à celle de la majorité des deux tiers, la commune de Fort-de-France voit son pouvoir diminuer au profit de la commune du Lamentin, tandis que le pouvoir de chacune des deux autres communes (Schœlcher, Saint-Joseph) reste inchangé ;

- dans le cas de CAP Nord, aucune commune ne détient à elle seule plus de la moitié du pouvoir. Sous chacune des règles de décision, c'est la commune du Robert qui détient le plus grand pouvoir. Lorsque l'on passe de la règle de la majorité simple à celle de la majorité des deux tiers, le pouvoir augmente pour les communes suivantes: Ajoupa-Bouillon, BassePointe, Bellefontaine et Gros-Morne. Les autres communes, à l'exception du Robert et de Trinité, voient leur indice de Shapley-Shubik diminuer alors que leur indice de BanzhafColeman augmente. La commune du Robert voit ses deux indices baisser alors que, pour la commune de Trinité, l'indice de Shapley-Shubik augmente et celui de Banzhaf-Coleman diminue ;

- dans le cas de la CAESM, nous avons noté plus haut que la distribution actuelle des sièges était différente de celle qu'aurait recommandée la loi. À cet effet, nous avons calculé les indices de pouvoir sous chacune des deux configurations : en rouge et entre parenthèses, les indices de pouvoir lorsque la distribution suit les recommandations de la loi. Si l'on s'en tient à la distribution des sièges actuelle, aucune commune ne dispose de plus de $12 \% \mathrm{du}$ pouvoir quelle que soit la règle de décision. Lorsque l'on passe de la règle de la majorité simple à celle de la majorité des deux tiers, aucune commune ne voit ses deux indices augmenter simultanément ; c'est plutôt l'effet inverse qui se produit pour cinq communes : Trois-Ilets, Marin, Saint-Esprit, Sainte-Luce et Vauclin. Pour les autres communes, les indices évoluent en sens opposés. Si l'on tenait plutôt compte de la distribution des sièges telle que recommandée par la loi, on obtiendrait un schéma quasi similaire. Lorsque l'on compare les configurations issues de ces deux distributions, il en ressort que si la règle de décision est celle de la majorité simple, comparativement à la distribution qu'aurait recommandée la loi, la distribution actuelle est favorable aux communes suivantes: Diamant, Anses-d'Arlet, Trois-Ilets, Rivière-Pilote, Rivière-Salée, Sainte-Anne ; si la règle de vote est la majorité des deux tiers, la distribution actuelle est défavorable aux communes suivantes : Diamant, Ducos, François, Trois-Ilets.

Nous avons procédé à des tests de Khi-deux (à $5 \%$ ) entre le pourcentage de population (de délégués) des communes et leurs indices de pouvoir ${ }^{15}$. L'objectif de ces tests est de répondre aux questions suivantes : Existe-t-il une similarité entre la distribution des indices de pouvoir et celle des pourcentages de population? Existe-t-il une similarité entre la distribution des indices de pouvoir et celle des pourcentages de délégués ? Les résultats de ces tests sont reportés dans la Table 7. 
Table 7. Tests de Khi-deux de similarité des distributions pour les EPCI de Martinique

\begin{tabular}{|l|l|l|l|l|l|}
\hline & \multicolumn{3}{|l|l|l|}{$\begin{array}{l}\text { Décision à la majorité } \\
\text { simple }\end{array}$} & $\begin{array}{l}\text { Décision à la majorité des } \\
\text { deux tiers }\end{array}$ \\
\hline $\begin{array}{l}\text { Communauté } \\
\text { d'agglomération }\end{array}$ & $\begin{array}{l}\text { Variable de } \\
\text { test }\end{array}$ & $\begin{array}{l}\text { Shapley- } \\
\text { Shubik }\end{array}$ & $\begin{array}{l}\text { Banzhaf- } \\
\text { Coleman }\end{array}$ & $\begin{array}{l}\text { Shapley- } \\
\text { Shubik }\end{array}$ & $\begin{array}{l}\text { Banzhaf- } \\
\text { Coleman }\end{array}$ \\
\hline \multirow{2}{*}{ CACEM } & \% Population & $\begin{array}{l}\text { Non } \\
(0,261)\end{array}$ & $\begin{array}{l}\text { Non } \\
(0,261)\end{array}$ & $\begin{array}{l}\text { Non } \\
(0,238)\end{array}$ & $\begin{array}{l}\text { Non } \\
(0,238)\end{array}$ \\
\cline { 2 - 6 } & \% Nb. Délégués & $\begin{array}{l}\text { Non } \\
(0,261)\end{array}$ & $\begin{array}{l}\text { Non } \\
(0,261)\end{array}$ & $\begin{array}{l}\text { Non } \\
(0,238)\end{array}$ & $\begin{array}{l}\text { Non } \\
(0,238)\end{array}$ \\
\hline \multirow{2}{*}{ CAESM } & \% Population & $\begin{array}{l}\text { Non } \\
(0,347)\end{array}$ & $\begin{array}{l}\text { Non } \\
(0,347)\end{array}$ & $\begin{array}{l}\text { Non } \\
(0,347)\end{array}$ & $\begin{array}{l}\text { Non } \\
(0,347)\end{array}$ \\
\cline { 2 - 6 } & \% Nb. Délégués & $\begin{array}{l}\text { Oui } \\
(0,000)\end{array}$ & $\begin{array}{l}\text { Oui } \\
(0,000)\end{array}$ & $\begin{array}{l}\text { Oui } \\
(0,000)\end{array}$ & $\begin{array}{l}\text { Oui } \\
(0,000)\end{array}$ \\
\hline Note. (.) p-valeur & \% Population & $\begin{array}{l}\text { Non } \\
(0,189)\end{array}$ & $\begin{array}{l}\text { Non } \\
(0,189)\end{array}$ & $\begin{array}{l}\text { Non } \\
(0,189)\end{array}$ & $\begin{array}{l}\text { Non } \\
(0,189)\end{array}$ \\
\hline
\end{tabular}

Source : calculs des auteurs

D'après la Table 7, on note qu'au sein de la CACEM, les distributions des indices de pouvoir ne sont pas similaires à celle de la population ni à celle du nombre de délégués. Ceci traduit le fait qu'au sein de cet EPCI l'indice de pouvoir des communes n'est équivalent ni à leurs poids démographiques ni à la proportion du nombre de délégués. $\mathrm{Au}$ sein de la CAESM et de CAP Nord, on note une certaine similitude entre les distributions des indices de pouvoir et celle du nombre de délégués; au contraire, les distributions des indices de pouvoir ne sont pas similaires à celle de la population.

\subsection{Le pouvoir des communes dans les $\mathrm{EPCl}$ guadeloupéens}

Les calculs des indices de pouvoir de chacune des communes de chacun des EPCI de la Guadeloupe sont consignés dans la Table 6 (en annexe). De la Table 6, nous déduisons que :

- au sein de CAP Excellence, lorsque l'on passe de la règle de la majorité simple à celle de la majorité des deux tiers, le rapport de force entre les communes reste le même. La commune des Abymes apparaît comme un joueur "pivot». On arrive exactement aux mêmes conclusions si l'on se base plutôt sur la répartition des délégués qu'aurait suggérée la loi ;

- au sein de la communauté d'agglomération La Riviera du Levant, lorsque la règle de décision est celle de la majorité simple, la commune de Désirade a un pouvoir nul tandis que les 
autres communes ont exactement le même pouvoir. Mais lorsqu'on passe à la majorité des deux tiers, la commune du Gosier détient plus de $50 \%$ de pouvoir de vote et les communes de Désirade et de Saint-François conservent le même pouvoir. Si l'on se fondait plutôt sur la répartition des délégués telle que suggérée par la loi, les rapports de force sous la majorité simple resteraient inchangés; tandis que sous la majorité des deux tiers, les communes de Désirade et de Saint-François n'auraient aucun pouvoir. Ainsi, si la règle de décision est celle de la majorité simple, la distribution actuelle ne favorise aucune commune en comparaison avec la répartition des délégués qu'aurait suggérée la loi. Tel est par contre le cas sous la majorité des deux tiers où les communes de Désirade et de Saint-François se trouvent avantagées ;

- au sein de la communauté d'agglomération du Nord Grande-Terre, la répartition actuelle est défavorable à la commune de Moule quelle que soit la règle de décision, aux communes de Petit-Canal et Port-Louis sous la règle de la majorité simple et à la commune de Morne-ÀL'eau sous la règle de la majorité des deux tiers.

- au sein de la communauté d'agglomération du Nord Basse-Terre, aucune commune ne dispose de plus de $40 \%$ de pouvoir de vote quelle que soit la règle de décision. Lorsqu'on passe de la règle de la majorité simple à celle de la majorité des deux tiers, les communes de Deshaies et de Pointe-Noire voient leur pouvoir quasiment doubler, celle de Goyave voit son pouvoir quasiment multiplié par quatre alors que les autres communes voient leur pouvoir diminuer ;

- au sein de la communauté d'agglomération du Sud Grande-Terre, aucune commune ne possède plus de $30 \%$ de pouvoir de vote. La répartition actuelle est défavorable aux communes de Basse-Terre et de Saint-Claude sous la règle de la majorité simple, et aux communes de Bouillante, Gourbeyre et de Trois-Rivières sous la règle de la majorité des deux tiers ;

- étant donné la répartition actuelle sein de la communauté de communes de Marie-Galante, chacune des trois communes détient exactement le même pouvoir de vote sous la règle de décision à la majorité simple. Sous la règle de la majorité des deux tiers, la commune de Grand-Bourg détient plus $60 \%$ de pouvoir, tandis que les deux autres communes ont le même pouvoir (20\%). Avec la répartition des délégués qu'aurait suggérée la loi, la commune de Grand-Bourg détiendrait entre 60 et $67 \%$ de pouvoir de vote quelle que soit la règle de décision, et les deux autres communes auraient le même pouvoir : 16,67 \% sous ShapleyShubik et $20 \%$ sous Shapley. On conclut que, sous la majorité des deux tiers, la distribution actuelle des pouvoirs est exactement égale à celle qu'on aurait obtenue si la loi avait été appliquée. Par contre, sous la majorité simple, la distribution désavantage la commune de Grand-Bourg au profit des deux autres communes.

Comme dans le cas des EPCI de la Martinique, nous avons procédé à des tests de Khideux entre le pourcentage de population (de délégués) des communes et leurs indices de pouvoir ${ }^{16}$. Les résultats de ces tests sont reportés dans la Table 8. D'après cette table, la distorsion entre les distributions est quasiment généralisée à tous les EPCI. L'exception provient de la distribution des indices de pouvoir et du pourcentage des délégués au sein de la communauté d'agglomération Grand Sud Caraïbe.

Table 8. Tests de Khi-deux de similarité des distributions

\begin{tabular}{|l|l|l|l|}
\hline & $\begin{array}{l}\text { Décision à la majorité } \\
\text { simple }\end{array}$ & $\begin{array}{l}\text { Décision à la majorité des } \\
\text { deux tiers }\end{array}$ \\
\hline
\end{tabular}




\begin{tabular}{|c|c|c|c|c|c|}
\hline $\begin{array}{l}\text { Communauté } \\
\text { d'agglomération }\end{array}$ & $\begin{array}{l}\text { Variable de } \\
\text { test }\end{array}$ & $\begin{array}{l}\text { Shapley- } \\
\text { Shubik }\end{array}$ & $\begin{array}{l}\text { Banzhaf- } \\
\text { Coleman }\end{array}$ & $\begin{array}{l}\text { Shapley- } \\
\text { Shubik }\end{array}$ & $\begin{array}{l}\text { Banzhaf- } \\
\text { Coleman }\end{array}$ \\
\hline \multirow{2}{*}{ CAP Excellence } & $\%$ Population & $\begin{array}{l}\text { Non } \\
(0,223)\end{array}$ & $\begin{array}{l}\text { Non } \\
(0,223)\end{array}$ & $\begin{array}{l}\text { Non } \\
(0,223)\end{array}$ & $\begin{array}{l}\text { Non } \\
(0,223)\end{array}$ \\
\hline & $\%$ Nb. Délégués & $\begin{array}{l}\text { Non } \\
(0,223)\end{array}$ & $\begin{array}{l}\text { Non } \\
(0,223)\end{array}$ & $\begin{array}{l}\text { Non } \\
(0,223)\end{array}$ & $\begin{array}{l}\text { Non } \\
(0,223)\end{array}$ \\
\hline \multirow{2}{*}{ La Riviera du Levant } & $\%$ Population & $\begin{array}{l}\text { Non } \\
(0,261)\end{array}$ & $\begin{array}{l}\text { Non } \\
(0,261)\end{array}$ & $\begin{array}{l}\text { Non } \\
(0,238)\end{array}$ & $\begin{array}{l}\text { Non } \\
(0,238)\end{array}$ \\
\hline & $\%$ Nb. Délégués & $\begin{array}{l}\text { Non } \\
(0,261)\end{array}$ & $\begin{array}{l}\text { Non } \\
(0,261)\end{array}$ & $\begin{array}{l}\text { Non } \\
(0,238)\end{array}$ & $\begin{array}{l}\text { Non } \\
(0,238)\end{array}$ \\
\hline \multirow{2}{*}{ CA Nord Grande-Terre } & $\%$ Population & $\begin{array}{l}\text { Non } \\
(0,241)\end{array}$ & $\begin{array}{l}\text { Non } \\
(0,241)\end{array}$ & $\begin{array}{l}\text { Non } \\
(0,287)\end{array}$ & $\begin{array}{l}\text { Non } \\
(0,287)\end{array}$ \\
\hline & $\% \mathrm{Nb}$. Délégués & $\begin{array}{l}\text { Non } \\
(0,241)\end{array}$ & $\begin{array}{l}\text { Non } \\
(0,241)\end{array}$ & $\begin{array}{l}\text { Non } \\
(0,287)\end{array}$ & $\begin{array}{l}\text { Non } \\
(0,287)\end{array}$ \\
\hline \multirow{2}{*}{ CA Nord Basse-Terre } & $\%$ Population & $\begin{array}{l}\text { Non } \\
(0,285)\end{array}$ & $\begin{array}{l}\text { Non } \\
(0,285)\end{array}$ & $\begin{array}{l}\text { Non } \\
(0,263)\end{array}$ & $\begin{array}{l}\text { Non } \\
(0,263)\end{array}$ \\
\hline & $\%$ Nb. Délégués & $\begin{array}{l}\text { Non } \\
(0,285)\end{array}$ & $\begin{array}{l}\text { Non } \\
(0,285)\end{array}$ & $\begin{array}{l}\text { Non } \\
(0,263)\end{array}$ & $\begin{array}{l}\text { Non } \\
(0,263)\end{array}$ \\
\hline \multirow{2}{*}{ CA Grand Sud Caraïbe } & $\%$ Population & $\begin{array}{l}\text { Non } \\
(0,291)\end{array}$ & $\begin{array}{l}\text { Non } \\
(0,291)\end{array}$ & $\begin{array}{l}\text { Non } \\
(0,291)\end{array}$ & $\begin{array}{l}\text { Non } \\
(0,291)\end{array}$ \\
\hline & $\%$ Nb. Délégués & $\begin{array}{l}\text { Oui } \\
(0,000)\end{array}$ & $\begin{array}{l}\text { Oui } \\
(0,000)\end{array}$ & $\begin{array}{l}\text { Oui } \\
(0,000)\end{array}$ & $\begin{array}{l}\text { Oui } \\
(0,000)\end{array}$ \\
\hline \multirow{2}{*}{ CC de Marie-Galante } & $\%$ Population & - & - & $\begin{array}{l}\text { Non } \\
(0,223)\end{array}$ & $\begin{array}{l}\text { Non } \\
(0,223)\end{array}$ \\
\hline & $\%$ Nb. Délégués & - & - & $\begin{array}{l}\text { Non } \\
(0,223)\end{array}$ & $\begin{array}{l}\text { Non } \\
(0,223)\end{array}$ \\
\hline
\end{tabular}

Source : calculs des auteurs

Comme on peut le constater, dans les Tables 7 et 8 , nous comparons plusieurs tests à un niveau donné (ici $5 \%$ ); notons qu'un tel exercice reste tout de même délicat. Néanmoins, il existe des méthodes statistiques pour contourner ce problème telles que les tests de Tukey, de Scheffé, de Bonferonni et de Newman-Keuls. Pour un aperçu et une comparaison de ces tests, le lecteur peut se référer aux travaux de Kleinbaum et al. (1998) et de Hsu (1996). 


\section{Conclusion} Martinique et de la Guadeloupe ont rejoint une intercommunalité dans laquelle chacune d'elle possède au moins un délégué. La proportion de délégués par commune n'étant pas toujours équivalente à la proportion de sa population dans l'EPCI, le pouvoir décisionnel et la représentativité d'une commune ne sauraient être approximés par le poids en délégués. Ce pouvoir peut être mesuré au moyen des indices de pouvoir de Shapley-Shubik et de Banzhaf-Coleman. L'application de ces indices aux EPCI de Martinique et de Guadeloupe nous a permis de mettre en évidence le fait que, dans certains EPCI, il existe des communes qui occupent le statut de "commune pivot» au sens où le vote des délégués de cette commune détermine l'issue de tout vote. Nous avons aussi noté que, dans quasiment tous les EPCI de Martinique et de Guadeloupe, il n'existe pas de similarité entre la distribution des indices de pouvoir et celle des pourcentages de population. On remarque par ailleurs une distorsion entre la distribution des indices de pouvoir et celle des pourcentages de délégués. Notre étude nous a également permis de constater une différence entre le nombre de délégués de certains EPCI et celui que recommande la loi de 2010 (Table 1). Aussi, nous avons noté qu'étant donné le nombre actuel de délégués dans certains EPCI, la répartition entre les communes n'obéit pas au mécanisme décrit par la loi. Ainsi, la répartition actuelle tend à favoriser certaines communes, ce qui se répercute dans les pouvoirs de vote. Une règle de décision est équitable si elle donne à chaque électeur le même pouvoir de vote. Dans un système indirect comme celui des EPCI, parler d'équité revient à dire que chaque habitant des communes au sein d'un EPCI possède le même pouvoir d'influencer les décisions collectives. Nos calculs témoignent bien du fait que c'est loin d'être une réalité pour les EPCI au cœur de cet article. Ce résultat est en accord avec les conclusions de Abidi, Le Prince et Merlin (2019), qui tendent à généraliser cette absence d'équité à un vaste ensemble d'EPCI de France métropolitaine.

Notons que des extensions du présent travail sont envisageables, notamment dans deux directions. À partir des travaux de Barthelemy et Martin (2007) et de Leech (2002), on pourrait envisager une analyse se fondant sur l'utilisation d'un test d'égalité des distributions basé sur des mesures de proximité telles que les distances. L'autre piste possible serait de reprendre notre approche méthodologique à partir d'un ensemble d'EPCI simulés tout en s'intéressant à des lois de probabilités proches des lois empiriques, comme c'est généralement le cas lorsqu'on procède au bootstrap.

\section{BIBLIOGRAPHIE}

Abidi Z., M. Le Prince \& V. Merlin, (2019), « Power Inequality in Inter-communal Structures: the Simulated Impact of a Reform in the Case of the Municipalities in Western France ", À paraître dans Économie et Prévision.

Économie et institutions, 28 | 2020 
Andjiga N.G., F. Chantreuil \& D. Lepelley, (2003), « La mesure du pouvoir de vote », Mathématiques et sciences humaines, $\mathrm{n}^{\circ}$ 163, p. 111-145 ; [En ligne], 163 | Automne 2003. URL : http:// journals.openedition.org/msh/2920 163 [consulté le 24/04/2020]

Balinski M. \& P. Young, (2001), Fair Representation: Meeting the Ideal of One Man, One Vote, Washington D.C, Brookings Institution Press, 2nd edition.

Banzhaf J.R, (1965), « Weighted Voting Doesn't Work: A Mathematical Analysis », Rutgers Law Review, 19(2), p. 317-343.

Barthelemy F. \& M. Martin, (2007), « Critères pour une meilleure répartition des sièges au sein des structures intercommunales : une application au cas du Val-d'Oise », Revue économique, 58(2), p. 399-425.

Barthelemy F. \& M. Martin, (2011), « A Comparison Between the Methods of Apportionment Using Power Indices: the Case of the US Presidential Elections ", Annals of Economics and Statistics, vol. 101/102, p. 87-106.

Barthelemy F., A. Béraud \& M. Martin, (2008), « Loi relative aux libertés et responsabilités des universités (loi LRU), élection du président et conseil d'administration : une analyse en termes de pouvoir ", Revue d'économie politique, (118)3, p. 299-315.

Barthelemy F., A. Béraud \& M. Martin, (2009), « La loi LRU a-t-elle modifié les distributions de pouvoir au sein des universités françaises », Revue économique, 60(6), p. 1469-1481.

Bisson F., J. Bonnet \& D. Lepelley, (2004), « La determination du nombre de délégués au sein des structures intercommunales : une application de l'indice de pouvoir de Banzhaf », Revue d'Économie Régionale \& Urbaine, $\mathrm{n}^{\circ}$ 2, p. 259-281.

Bobay F., (2001), « La réforme du Conseil de l'Union européenne à partir de la théorie des jeux », Revue française d'économie, 16(2), p. 3-61.

Bobay F., (2004), « Constitution européenne : redistribution du pouvoir des États au Conseil de l'UE », Économie \& prévision, 163(2), p. 101-115.

Bonnet J. \& D. Lepelley, (2001), « Pouvoir de vote et Intercommunalité : le cas des Établissements publics de coopération intercommunale de la région de Basse-Normandie », in R. Le Duff, J.P. Rigal \& G. Schmidt (dir.), Démocratie et management local, Paris, Dalloz, p. 519-532.

Coleman J.S., (1971), « Control of Collectivites and the Power of a Collectivity to Act », in B. Lieberman (ed.), Social Choice, New York, Gordon and Breach, p. 269-300.

Diss M. \& F. Steffen, (2018), « The Distribution of Power in the Lebanese Parliament Revisited », Working Paper No 1723, GATE Lyon Saint-Étienne.

Diss M. \& A. Zouache, (2015), « Une étude de la répartition du pouvoir confessionnel au Liban », Revue d'économie politique, 125(4), p. 527-546.

Felsenthal D.S. \& M. Machover, (1998), The Measurement of Voting Power: Theory and Practice, Problems and Paradoxes, Cheltenham (UK) \& Northampton (US), Edward Elgar.

Felsenthal D.S. \& M. Machover, (2001), « The Treaty of Nice and Qualified Majority Voting », Social Choice and Welfare, $\mathrm{n}^{\circ} 18$, p. 431-464.

Felsenthal D.S. \& M. Machover, (2003), « Analysis of QM Rules in the Draft Constitution for Europe Proposed by the European Convention », Social Choice and Welfare, $\mathrm{n}^{\circ} 23, \mathrm{p} .1-20$.

Garrett G. \& G. Tsebelis, (1999), « Why Resist the Temptation to Apply Power Indices to European Union? », Journal of Theoretical Politics, 11(3), p. 291-308. 
Herne K. \& H. Nurmi, (1993), « The Distribution of a priori Voting Power in the EC Council of Ministers and the European parliament », Scandinavian Political Studies, 16(3), p. 269-284.

Holler M. \& H. Nurmi, (2013), Power, Voting, and Voting Power: 30 Years After, Berlin \& New York, Springer.

Hsu J.C., (1996), Multiple Comparisons: Theory and Methods, Boca Raton, Chapman and Hall.

Kleinbaum D.G, L.L. Kupper, A Nizam \& E.S. Rosenberg, (1998), Applied Regression Analysis and Other Multivariable Methods, (3rd edition), Pacific Grove, Duxbury Press.

Laruelle A. \& M. Wigrén, (1998), « Is the Allocation of Voting Power among EU States Fair? », Public Choice, $\mathrm{n}^{\circ}$ 94, p. 317-339.

Leech D., (2002), « Designing the Voting System for the Council of the European Union », Public Choice, vol. 113, p. 437-464.

Leech D. \& H. Aziz, (2010), « The Double Majority Voting Rule of the EU Reform Treaty as a Democratic Ideal for an Enlarging Union: an Appraisal Using Voting Power Analysis ", in M.A. Cichocki \& K. Życzkowski (eds), Institutional Design and Voting Power in the European Union, Farnham (UK), Ashgate Publishing.

Leech D. \& R. Leech, (2016), « Computer Algorithms for Voting Power Analysis ». URL: http:// homepages.warwick.ac.uk/ ecaae/

Pajala A., T. Meskanen \& T. Kause, (2002), « Powerslave Power Index Calculator: A Voting Body Analyser in the Voting Power and Power Index Website », University of Turku. URL: http:// powerslave.val.utu.fi/ (Updated 31.5.2007)

Penrose L.S., (1946), « The Elementary Statistics of Majority Voting », Journal of the Royal Statistical Society, 109(1), p. 53-57.

Penrose L.S., (1952), On the objective study of crowd behavior, London, H.K. Lewis.

Shapley L.S \& M. Shubik, (1954), « A Method for Evaluating the Distribution of Power in a Committee System », American Political Science Review, 48(3), p. 787-792.

Straffin P.D. (Jr.), (1977), « Homogeneity, Independence and Power Indices », Public Choice, $\mathrm{n}^{\circ}$ 30, p. 107-118.

\section{NOTES}

1. Voir Portraits régionaux de l'intercommunalité, départements et régions d'outre-mer. URL : https:// drive.google.com/open?id= 1wfx0vUsQbGTCimBlCfzFR5pqDf7pKzo-

2. Centre national de la fonction publique territoriale. URL : http://www.cnfpt.fr/sites/default/ files/intercommunalite.pdf

3. Les syndicats de communes, les communautés de communes, les communautés d'agglomération, les communautés urbaines, les syndicats mixtes et les métropoles.

4. Syndicat intercommunal à vocation unique.

5. Syndicat intercommunal à vocation multiple.

6. Voir la Table 2 en annexe pour la liste des communes constitutives des EPCI de la Martinique.

7. Voir la Table 3 en annexe pour la liste des communes constitutives des EPCI de la Guadeloupe.

8. Anciennement, communauté d'agglomération du Sud Basse-Terre (CASBT) entre 2012 et 2014.

9. Cette loi fut appliquée pour la première fois lors des élections municipales de 2014. 
10. Encore appelé indice de Banzhaf normalisé.

11. Loi relative aux libertés et responsabilités des universités.

12. Il est supposé qu'aucun agent ne s'abstient.

13. Notons qu'il existe d'autres logiciels ou interfaces qui permettent le calcul des différents indices de pouvoir. Le lecteur peut à ce propos consulter Leech et Leech (2016).

14. Code général des collectivités territoriales.

15. Ici, nos calculs sont basés sur les distributions actuelles.

16. Les calculs sont basés sur les distributions actuelles.

\section{RÉSUMÉS}

Cet article s'intéresse à la répartition des sièges entre les communes dans les établissements publics de coopération intercommunale (EPCI) de la Martinique et de la Guadeloupe. Dans chacun des EPCI, nous calculons le pouvoir de vote des communes au moyen des indices de ShapleyShubik et de Banzhaf-Coleman. Notre analyse nous permet de montrer que, dans quasiment tous les EPCI de la Martinique et de la Guadeloupe, il n'existe pas de similarité entre la distribution des indices de pouvoir et celle des pourcentages de population. On note aussi une distorsion entre la distribution des indices de pouvoir et celle des pourcentages de délégués.

This paper deals with the distribution of seats between municipalities in the inter-communal council (EPCI) of Martinique and Guadeloupe. In each of the EPCIs, we calculate the voting power of municipalities using the Shapley-Shubik and Banzhaf-Coleman indices. Our analysis allows us to show that in almost all the EPCIs of Martinique and Guadeloupe, there is no similarity between the distribution of power indices and the distribution of population percentages. There is also a distortion between the distribution of power indices and the distribution of delegate percentages.

\section{INDEX}

Mots-clés : Banzhaf-Coleman, indice de pouvoir, intercommunalité, Shapley- Shubik, vote

Keywords : Banzhaf-Coleman, municipalities, Shapley- Shubik, vote, power index

\section{AUTEURS}

\section{IBRAHIMA DIA}

Université de Guyane, DFR Sciences Juridiques et Economiques. Chercheur associé au Centre de Recherche en Économie et Management, CREM UMR CNRS 6211. Email : ibrahima.dia@univunicaen.fr 


\section{ÉRIC KAMWA}

Université des Antilles, Faculté de Droit et d'Économie, Campus de Schœlcher. Laboratoire Caribéen de Sciences Sociales, LCSS UMR CNRS 8053. Chercheur associé au Centre de Recherche en Économie et Management, CREM UMR CNRS 6211. Email : eric.kamwa@univ-antilles.fr 